\title{
AICAR Sustains J1 Mouse Embryonic Stem Cell Self-Renewal and Pluripotency by Regulating Transcription Factor and Epigenetic Modulator Expression
}

\author{
Xiaoyan Shi ${ }^{a, c}$ Yongyan Wu ${ }^{b, c}$ Zhiying Aia,c Xin Liu ${ }^{a, c}$ Liping Yang ${ }^{a, c}$ Juan Du ${ }^{a, c}$ \\ Jingjing Shao ${ }^{a, c}$ Zekun Guo ${ }^{b, c}$ Yong Zhang ${ }^{b, c}$
}

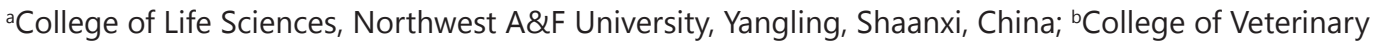
Medicine, Northwest A\&F University, Yangling, Shaanxi, China; 'Key Laboratory of Animal Biotechnology, Ministry of Agriculture, Northwest A\&F University, Yangling, Shaanxi, China

\section{Key Words}

Small molecule • Pluripotency • Gene expression • Signal transduction • Epigenetic

\begin{abstract}
Backgroud/Aims: Embryonic stem cells (ES cells) have the capacity to propagate indefinitely, maintain pluripotency, and differentiate into any cell type under defined conditions. As a result, they are considered to be the best model system for research into early embryonic development. AICA ribonucleotide (AICAR) is an activator of AMP-activated protein kinase (AMPK) that is thought to affect ES cell function, but its role in ES cell fate decision is unclear. Methods: In this study, we performed microarray analysis to investigate AICAR downstream targets and further understand its effect on ES cells. Results: Our microarray data demonstrated that AICAR can significantly up-regulate pluripotency-associated genes and down-regulate differentiation-associated transcription factors. Although AICAR cannot maintain ES cell identity without LIF, it can antagonize the action of RA-induced differentiation. Using those differentially expressed genes identified, we performed gene ontology (GO) and Kyoto Encyclopedia of Genes and Genomes (KEGG) pathway analysis with the Database for Annotation, Visualization and Integrated Discovery (DAVID) online system. AICAR was not only shown to influence the AMPK pathway, but also act on other signaling pathways such as BMP, MAPK and TGF- $\beta$, to maintain the stemness of J1 ES cells. Furthermore, AICAR modulated ES cell epigenetic modification by altering the expression of epigenetic-associated proteins, including Dnmt3a, Dnmt3b, Smarca2, Mbd3, and Arid1a, or through regulating the transcription of long intervening non-coding RNA (lincRNA). Conclusion: Taken together, our work suggests that AICAR is capable of maintaining ES cell self-renewal and pluripotency, which could be useful in future medical treatment.
\end{abstract}


Shi et al.: AICAR Modulates mESCs Gene Expression

\section{Introduction}

Embryonic stem cells (ES cells) are totipotent stem cells derived from the inner cell mass of the blastocyst $[1,2]$. Their distinctive properties include indefinite replication under defined conditions, self-renewal and pluripotency [3], in that they can differentiate into almost any cell type in the body, both in vitro and in vivo [4]. They also have the ability to undergo numerous cycles of cell division while maintaining their undifferentiated state. Because of these properties, ES cells are considered an excellent model system to study the fundamental biology of early embryonic development, tissue homeostasis and genetic disease. They are also expected to be used in treatment such as regenerative medicine and tissue replacement. Therefore, investigations into the mechanisms and methods to maintain cellular pluripotency and direct cell fate are crucial to their application in the clinic.

It has been shown that small molecules can manipulate the fate of ES cells by promoting self-renewal or inhibiting the differentiation process $[5,6]$. Moreover, the effects of small molecules are reversible and adjustable compared to conventional genetic manipulation, because their concentration and combination can be fine-tuned according to requirements. Pluripotin/SC1 was the first identified molecule to maintain long-term self-renewal and ES cell pluripotency under defined conditions by inhibiting MEK-ERK and Ras-GAP differentiationinducing signaling pathways [7]. CHIR99021 was also found to enable ES cell self-renewal under feeder-free conditions through inhibition of GSK3, thus further activating the Wnt/ $\beta$-catenin pathway [8]. Other small molecules that can modulate the ES cell fate include glycogen synthase kinase 3 (GSK3) inhibitor BIO, MAPK/ERK kinase inhibitor PD0325901 and TGF- $\beta 1$ receptor ALK5 inhibitor SB431542 [9-11], which maintain pluripotency as well as elevating induced pluripotent stem cells (iPSCs)efficiency.

AICA ribonucleotide (AICAR) is an AMP analog capable of stimulating AMP-dependent protein kinase (AMPK) activity [12] and having diverse effects on glucose and lipid metabolism, protein synthesis, and cytokine production [13-15]. In medical usage, AICAR has been a potential treatment for cardiac ischemia and diabetes $[16,17]$. Moreover, it can trigger a preconditioned anti-inflammatory state to prevent post-ischemic leukocyte-endothelial cell adhesive interactions. Recent research showed that AICAR activates the ES cell p53/ p21 pathway and significantly reduces expression of pluripotent markers Nanog and stagespecific embryonic antigen-1 (SSEA-1) [18]. Induction of neural stem cell (NSC) astroglial differentiation by AICAR through JAK/STAT3 was also reported [12]. Conversely, others have shown that AICAR upregulates Nanog, Oct4, Klf4 and Myc expression and activates the pluripotency transcriptional network in mouse ES cells (mESCs) [19]. Nevertheless, AICAR modulation of ES cell global gene expression has not yet been elucidated. To investigate the mechanisms by which AICAR affects ES cells, we performed microarray analysis to identify differentially expressed genes in J1 ES cells cultured in ES cell medium with or without AICAR.

\section{Materials and Methods}

\section{Cell Culture}

J1 mouse ES cells were purchased from ATCC (Manassas, VA, USA). They were maintained at $37^{\circ} \mathrm{C}$ humidified air with $5 \% \mathrm{CO}_{2}$ on $0.2 \%$ gelatin-coated plates in knockout Dulbecco's modified Eagle's medium (DMEM) supplemented with 15\% KnockOut ${ }^{\mathrm{TM}}$ Serum Replacement, 2 mM glutamine, 0.1 mM nonessential amino acids, $125 \mu \mathrm{g} / \mathrm{ml}$ penicillin, $100 \mu \mathrm{g} / \mathrm{ml}$ Streptomycin, $0.1 \mathrm{mM} \beta$-mercaptoethanol, and 1,000 U/ml LIF (ESGRO, Millipore, Billerica, MA, USA). For gene expression analysis, J1 ES cells were passaged every two days to maintain an undifferentiated state. Cells were passaged by treating them with $0.25 \%$ trypsin and $0.02 \%$ EDTA to generate a single-cell suspension; trypsin activity was stopped with an equal volume of J1 cell medium. Cells were centrifuged at $1,000 \times g$ for $5 \mathrm{~min}$ and re-suspended in ES cell medium. Suspended cells were then plated on gelatin-coated $60 \mathrm{~mm}$ cell plates, 6 -well cell plates or 24-well cell plates according to experimental requirements. All cell culture reagents were purchased from Gibco (Invitrogen, Carlsbad, 
Shi et al.: AICAR Modulates mESCs Gene Expression

CA, USA) unless indicated and sterile cell wells were purchased from Nunclon (Roskilde, Denmark).

\section{AICAR and RA Treatment}

AICAR (Beyotime Institute of Biotechnology, Jiangsu, China), Dorsomorphin (Dorso, Sigma Chemical Co., Louis, MO, USA) and RA ( Sigma Chemical Co., Louis, MO, USA) were dissolved in dimethyl sulfoxide (DMSO) at a final concentration of $250 \mathrm{mM}, 1 \mathrm{mM}$ and $2 \mathrm{mM}$ respectively. AICAR was added to cell medium at a final concentration of $1 \mathrm{mM}$ for $24 \mathrm{~h}$, when seeded J1 ES cells achieved $50-60 \%$ confluency. Cell medium with an equal volume of DMSO was used as a control. The working concentration of RA was $2 \mu \mathrm{M}$. Dorso was used at a final concentration of $5 \mu \mathrm{M}$.

\section{RNA Isolation and Whole-genome Gene Expression Profiling of J1 ESCs}

Total RNA was extracted using TRIZOL Reagent (Life Technologies, Carlsbad, CA, USA) following the manufacturer's instructions. Total RNA samples were analyzed with Agilent SurePrint G3 Mouse GE 8*60K Microarray (Agilent technologies) by the Shanghai Biochip Company (Shanghai, China). Data were annotated using the SAS Analysis System and filtered by signal intensity and detection call (present (P), marginal (M), or absent (A)). Gene expression fold changes were compared between AICAR-treated and DMSO-treated J1 ES cells.

Microarray data were deposited in the NCBI GEO database (http://www.ncbi.nlm.nih.gov/geo/query/ acc.cgi? acc $=$ GSE40959).

\section{GO and KEGG Pathway Analysis}

To perform gene ontology (GO) and Kyoto Encyclopedia of Genes and Genomes (KEGG) pathway analysis, data selection was performed on the basis of gene expression fold change (FC $\geq 2$ or FC $\leq 0.5)$ and statistical significance $(p \leq 0.01)$.

GO analysis was performed using the database for Annotation, Visualization and Integrated Discovery Bioinformatics Resources (DAVID) (http://david.abcc.ncifcrf.gov/) with biological process FAT, cell component FAT and molecular function FAT datasets. To reduce the expected proportion of false positives among all significant hypothetical terms, we selected GO terms using an FDR threshold of $\leq 0.05$. The $p$-value represents the significance of genes in a particular GO term. Assuming that the total numbers of genes and differentially expressed genes are consistent, the more of genes fell in a particular GO term, the smaller the $p$-value and the more significant the event.

We also performed KEGG pathway analysis using the DAVID online program with the thresholds of count $\geq 4$ and EASE $<0.05$. In this performance, count is the number of genes belonging to an annotation pathway and the EASE score is a modified Fisher's exact $p$-value for gene-enrichment analysis. The $p$-value $\leq 0.05$ is usually considered to be strongly enriched in the annotation categories.

\section{RT-PCR and Quantitative Real-time PCR Analysis}

Validation of the Agilent microarray gene expression data was performed using quantitative real-time PCR analysis with the ABI StepOnePlus PCR system (Applied Biosystems, Foster City, CA, USA). Total RNA $(1 \mu \mathrm{g})$ was reverse transcribed using the PrimeScript ${ }^{\circledast}$ RT reagent Kit (TaKaRa, Dalian, China). Real-time PCR was performed in triplicate for each sample using the SYBR ${ }^{\circledR}$ Premix Ex Taq ${ }^{\mathrm{TM}}$ II (Perfect Real Time) kit (TaKaRa). All reactions were performed at $95^{\circ} \mathrm{C}$ for $30 \mathrm{~s}$, followed by 40 cycles of $95^{\circ} \mathrm{C}$ for $5 \mathrm{~s}$ and $60^{\circ} \mathrm{C}$ for 30 $\mathrm{s}$, ending with a melting curve acquisition. We used the housekeeping gene GAPDH to normalize the amount of template added. The $2^{-\Delta C T}$ method was used to analyze relative changes in gene expression, and samples treated by DMSO were used as a reference. The primer sequences used for real-time PCR are provided upon requirement.

\section{Dual Luciferase Reporter Assay}

Pathway reporter vectors pNFkB-TA-luc, pMyc-TA-luc and pAP1-TA-luc were purchased from Beyotime Institute of Biotechnology; pISRE-TA-luc, pAP1(PMA)-TA-luc and the negative control pTA-luc were purchased from Clontech Laboratories, Inc. (Mountain View, CA, USA). Other signaling transduction reporter vectors including pCRE-TA-luc, pGRE-TA-luc, pHSE-TA-luc and pSRE-TA-luc were constructed in our laboratory by inserting their cis-acting DNA binding sequence into the multiple cloning sites of pTA-luc. The inserted sequences were as follows: cAMP response element (CRE, GCA CCA GAC AGT GAC GTC AGC 
Shi et al.: AICAR Modulates mESCs Gene Expression

TGC CAG ATC CCA TGG CCG TCA TAC TGT GAC GTC TTT CAG ACA CCC CAT TGA CGT CAA TGG GAG AAC); Glucocorticoid Response Element (GRE, GGT ACA TTT TGT TCT AGA ACA AAA TGT ACC GGT ACA TTT TGT TCT); Heatshock response element (HSE, CTA GAA TGT TCT AGA TCT AGA ACA TTC TAG CTA GAA TGT TCT AGA); Serum Response Element (SRE, GAT GTC CAT ATT AGG ACA TCG ATG TCC GAA TAT GGA CAT CGA TGT CCA TAT TAG GAC ATC).

The negative control pTA-luc containing a TATA box from the herpes simplex virus thymidine kinase (HSV-TK) promoter was used to determine background signals, and pRL-SV40 (Promega, Madison, WI, USA) was used as an internal control to distinguish differences in transfection efficiency. Luciferase assays were performed with the Dual-Luciferase Reporter Assay System (Promega) according to the manufacturer's instructions. Briefly, J1 ES cells were seeded on gelatin-coated 24-well cell plates the day before transfection. When the cells obtained 50-60\% confluency, pathway reporter vectors driving the expression of firefly luciferase, and pRL-SV40 driving the expression of Renilla luciferase were cotransfected by Lipofectamine 2000 (Invitrogen) according to the manufacturer's protocol. $24 \mathrm{~h}$ after transfection, $1 \mathrm{mM}$ AICAR or an equal volume of DMSO was added to cell medium for another $24 \mathrm{~h}$. Cells were then lysed in $1 \times$ passive lysis buffer and luciferase activity was measured on a VICTOR X5 Multilabel Plate Reader (PerkinElmer, Norwalk, CT, USA).

\section{Immunofluorescence Staining}

J1 ES cells were treated with DMSO or AICAR for $24 \mathrm{~h}$ on gelatin-coated 24-well plates. The medium was then removed and cells were washed twice with PBS before being fixed and permeabilized with Immunol Staining Fix Solution for 10 min and blocked with Immunol Staining Blocking Buffer for 1 h. Cells were then incubated with Immunol Staining Primary Antibody Dilution Buffer-diluted primary antibodies overnight at $4^{\circ} \mathrm{C}$ and Immunol Fluorence Staining Secondary Antibody Dilution Buffer-diluted secondary antibodies conjugated with DyLight 549 for $2 \mathrm{~h}$ at room temperature in the dark. After each step, cells were washed three times with Immunol Staining Wash Buffer for 5 min before the next step. DAPI staining was performed after secondary antibody incubation for $10 \mathrm{~min}$ at room temperature. Cells were observed and photographed under a confocal microscope system (Nikon, Tokyo, Japan). The primary antibodies and dilutions used were: rabbit anti-KLF4 (1:50), mouse anti-H3K9me2 (1:500), rabbit anti-H3K9me3 (1:500), mouse anti-H3K4me3 (1:500), rabbit anti-H3K27me3 (1:500), mouse anti-5mC (1:500), rabbit anti-H3K9Ac (1:500) (all from Abcam, Cambridge, UK), mouse anti-Oct4 (Santa Cruz, CA, USA; 1:500), mouse anti-Sox2 (Santa; 1:500), rabbit anti-5hmC (Active Motif, Carlsbad, CA, USA, 1:500), and rabbit anti-Nanog (Bethyl Laboratories, Montgomery, TX, USA; 1:500). All reagents not indicated were purchased from the Beyotime Institute of Biotechnology.

Alkaline Phosphatase Activity Assay

J1 ES cells were seeded on gelatin-coated 6-well plates with AICAR or DMSO in the presence of 1,000 $\mathrm{U} / \mathrm{ml}$ LIF. After $24 \mathrm{~h}$, the alkaline phosphatase activity of cells cultured in defined medium was detected with the BCIP/NBT alkaline phosphatase color development kit (Beyotime Institute of Biotechnology) following the manufacturer's instructions.

\section{Statistical analysis}

Data were presented as the mean \pm standard deviation (SD), and statistical significances were analyzed using the Student's t-test. A value of $\mathrm{p}<0.05$ was considered significant.

\section{Results}

AICAR Combined with LIF Maintains J1 ES Cell Colony Morphology and Elevates Expression Levels of Core Pluripotent Stem Cell Markers

High expression of stem cell marker genes and positive of alkaline phosphatase activity are critical properties of ESCs [20]. To investigate the effects of AICAR on these properties of J1 ES cells, we cultured cells with 1,000 U/ml LIF and either $1 \mathrm{mM} \mathrm{AICAR}$ or an equal volume of DMSO for $24 \mathrm{~h}$. In the presence of AICAR, J1 ES cells showed high alkaline phosphatase activity, while those cultured without AICAR appeared with low alkaline phosphatase activity (Fig. 1a). 


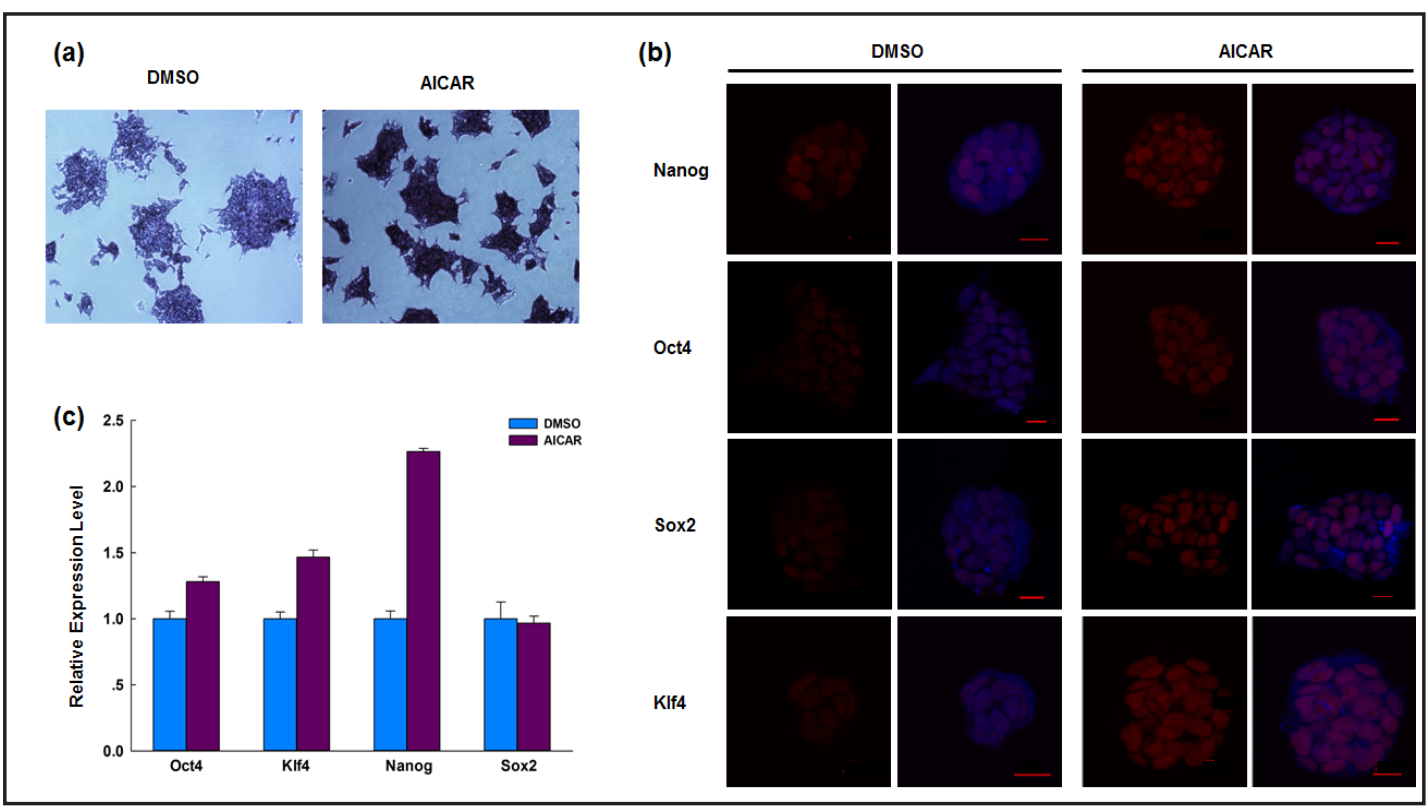

Fig. 1. AICAR combined with LIF maintains J1 mESC stemness under feeder-free conditions. (a): Alkaline phosphatase staining of $1 \mathrm{mESC}$ cultured on gelatin-coated plates in DMEM with knockoutserum replacement supplemented with 1,000 U/ml LIF, with or without $1 \mathrm{mM}$ AICAR for $24 \mathrm{~h}$. (b): Immunofluorescence staining of pluripotency markers Oct4, Klf4, Nanog and Sox2 in J1 mESCs in the presence of 1,000 U/ml LIF with or without $1 \mathrm{mM}$ AICAR for $24 \mathrm{~h}$. Cell nuclei were stained by DAPI. Left columns represent antibody staining of each marker and right columns represent merged image with DAPI staining. Scale bars represent $20 \mu \mathrm{m}$. (c): Real-time PCR validation of Oct4, Klf4, Nanog and Sox2 in J1 mESCs in the presence of 1,000 U/ml LIF with or without $1 \mathrm{mM}$ AICAR for $24 \mathrm{~h}$ using the comparative Ct method. GAPDH was used to normalize template levels. Data are presented as the mean \pm SD of three independent experiments.

Oct4, Klf4, Nanog and Sox2 are core pluripotent ES cell markers that are highly expressed in ES cells and are commonly used to induce iPSCs [21-24]. In this study, we performed immunofluorescence staining and real-time PCR to examine their expression level. We found that AICAR treatment can elevate the expression level of all four ES cell markers (Fig. 1b). Consistent with these results, real-time PCR showed increased expression of Oct4, Klf4 and Nanog, although Sox2 expression appeared unchanged (Fig. 1c). Notably, the fold change of these factors did not exceed 2, perhaps because of the strictly controlled level of main pluripotency factors in ESCs that limits disruption of normal regulation and prevents cell differentiation.

Therefore, these results indicated that AICAR combined with LIF can maintain J1 ES cell colony morphology, increase its alkaline phosphatase activity and elevate expression levels of core pluripotent stem cell markers.

Whole-genome Gene Expression Profiling Indicates that AICAR Maintains ES cells in a Pluripotent State

To establish how AICAR affects the ES cell fate, we performed gene expression microarray analysis of J1 ES cells cultured with or without AICAR and compared differentially expressed genes. In the presence of $1 \mathrm{mM}$ AICAR, we observed 1,346 genes significantly up-regulated ( $F C \geq 2, p \leq 0.01$ ) and 1,119 genes significantly down-regulated compared with those cultured in an equal volume of DMSO ( $\mathrm{FC} \leq 0.5, \mathrm{p} \leq 0.01$ ).

From the gene expression pattern (Fig. 2a), we found that besides the well-known pluripotency-associated genes identified above (Oct4, Klf4, and Nanog; Fig. 1b and 1c), other pluripotency-related genes such as Prdm14, Esrrb, Dppa5a, Tcl1, and Tcfcp211 were also upregulated in J1 ES cells after AICAR treatment. Prdm14 is a PR-domain zinc finger protein 
Fig. 2. Wholegenome gene expression profiling indicates that AICAR maintains mESCs in a pluripotent state. (a): Expression pattern of representative pluripotency- or differentiationassociated genes identified by microarray data in J1 mESCs cultured with or without 1 mM AICAR. High expression levels are shown in green and low in red. (b): Representative pluripotencyrelated genes, up-regulated in the presence of $1 \mathrm{mM}$ AICAR, were validated by real-time PCR. J1 mESCs were cultured in medium
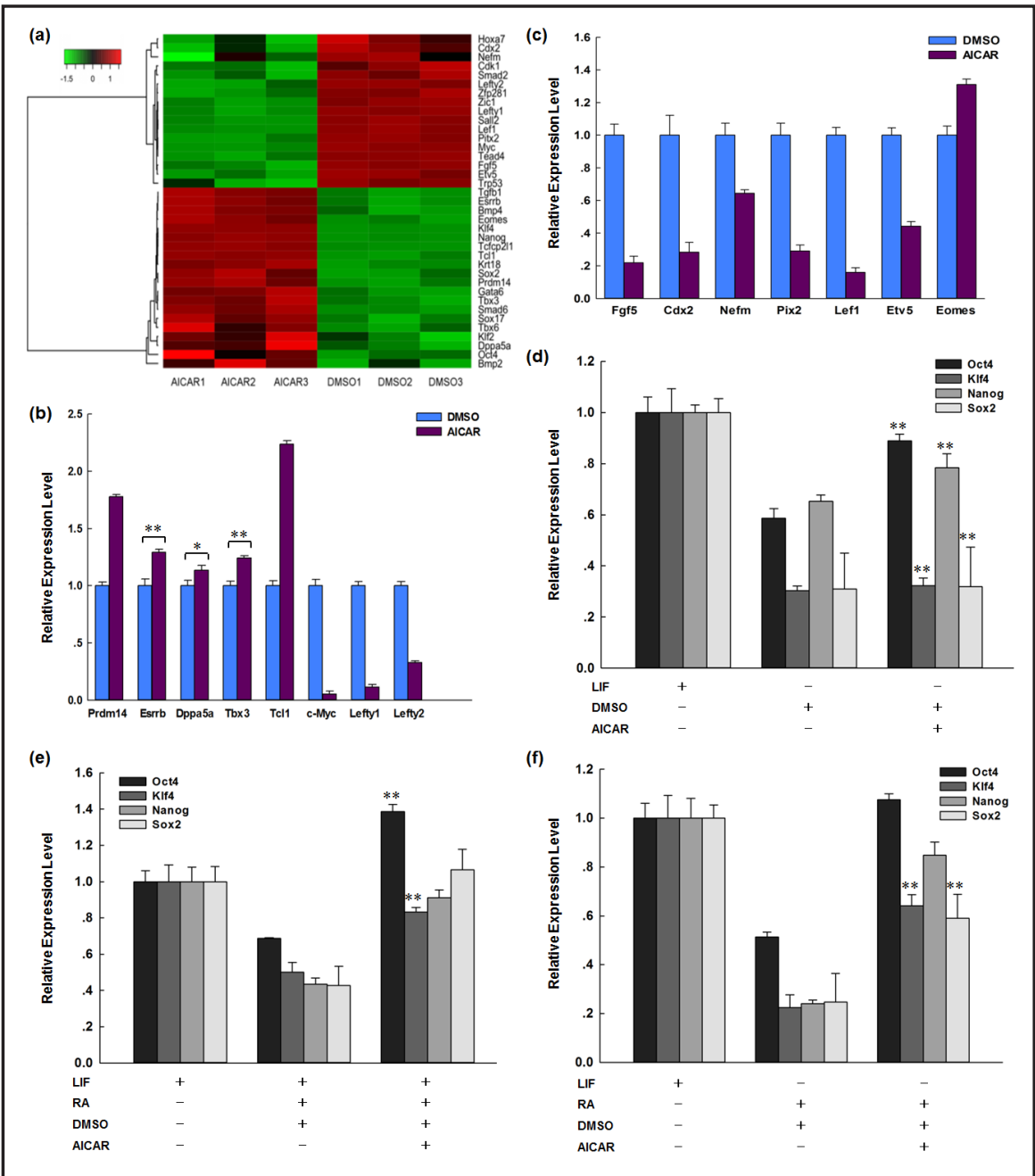

with serum and 1,000 U/ml LIF in the presence or absence of $1 \mathrm{mM}$ AICAR for $24 \mathrm{~h}$. Total RNA was isolated, and the relative expression level of genes determined by real-time PCR. Data are presented as the mean \pm SD of three independent experiments. (c): Representative differentiation-associated genes, down-regulated in the presence of $1 \mathrm{mM}$ AICAR, were validated by real-time PCR. J1 mESCs were cultured in medium containing serum and 1,000 U/ml LIF in the presence or absence of $1 \mathrm{mM}$ AICAR for $24 \mathrm{~h}$. Total RNA was isolated, and the relative expression level of genes determined by real-time PCR. GAPDH was used to normalize template levels. Data are presented as the mean \pm SD of three independent experiments. (d): Real-time PCR analysis of Oct4, Klf4, Nanog and Sox2 in J1 mESCs cultured in the absence of LIF and with or without AICAR for $24 \mathrm{~h}$ using the comparative Ct method. Cells cultured in normal medium with LIF were used as control. GAPDH was used to normalize template levels. Data are presented as the mean \pm SD of three independent experiments. (e): J1 ES cells were cultured in the presence of LIF and $2 \mu \mathrm{M}$ RA with or without AICAR for $24 \mathrm{~h}$; cells cultured in normal medium with LIF were used as control. The expression levels of Oct4, Klf4, Nanog and Sox2 were examined by real-time PCR. GAPDH was used to normalize template levels. Data are presented as the mean \pm SD of three independent experiments. (f): J1 ES cells were cultured in the absence of LIF, in presence of $2 \mu \mathrm{M}$ RA and with or without AICAR for $24 \mathrm{~h}$, cells cultured in normal medium with LIF were used as control. The expression levels of Oct4, Klf4, Nanog and Sox2 were examined by real-time PCR. GAPDH was used to normalize template levels. Data are presented as the mean \pm SD of three independent experiments. $(*: \mathrm{p}<0.05 ; * *: \mathrm{p}<0.01)$.

that maintains mESC pluripotency by preventing them from extra-embryonic endoderm (ExEn) fates. It can bind to and promote the expression of mESC self-renewal-associated 
Fig. 3. AICAR regulates multiple GO term and signaling transduction pathways. (a): The GO term comparison of differently expressed genes identified by gene expression microarray. The DAVID online system analyzed 1,346 significantly up-regulated genes and 1,119 significantly down-regulated genes identified by gene expression microarray for GO classification. Data of biological process (BP) FAT, cellular component (CC) FAT and molecular function (MF) FAT identified are shown as a $-\log P$ value and presented in a bar chart. (b): Dual luciferase reporter assay to identify signaling transduction pathways regulated by AICAR. Pathway reporter vectors (including negative control) and internal control pRL-SV40 were cotransfected by Lipofectamine 2000. $24 \mathrm{~h}$ after transfection, 1 mM AICAR or an equal volume of

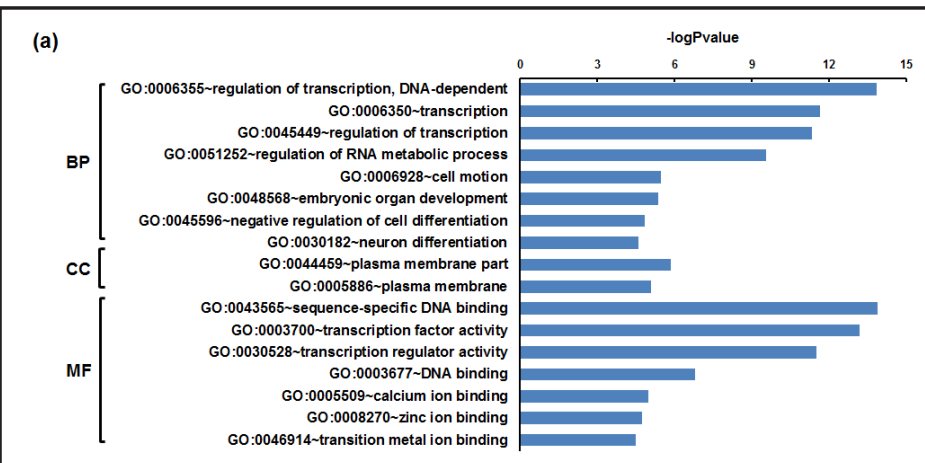

(b)

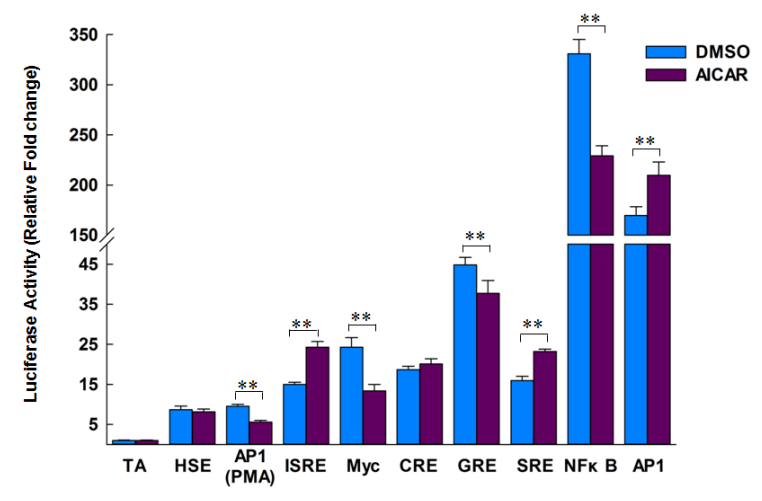

DMAO was added to cell medium for another $24 \mathrm{~h}$. Luciferase activity is presented relative to negative control pTA-luc. Data are presented as the mean \pm SD of three independent experiments. $(*: \mathrm{p}<0.05 ; * *: \mathrm{p}<0.01)$.

genes [25]. Esrrb (estrogen-related-receptor $\beta$ ) is a member of the nuclear orphan receptor family, which coordinates with Nanog and Oct4 to sustain self-renewal and pluripotency in ES cells [26]. Tcl1 can be activated by Oct3/4 and is involved in the regulation of ES cell proliferation [27], while Tcfcp2l1 is a member of the CP2 family and plays a critical role in the maintenance of ES cells. Expression of these up-regulated genes was validated by realtime PCR, and the results were consistent with our microarray data (Fig. 2b).

On the other hand, genes associated with development or tissue formation, such as Etv5, Fgf5, Cdx2, Nefm, Pitx2, Lef1, Trp53, Zic1, Hoxa7, and Sall2 (Fig. 2a), were significantly down-regulated. Fgf5 is a member of the fibroblast growth factor (FGF) family whose expression is up-regulated during ES cell differentiation [28]. Cooperating with Brachyury, $\mathrm{Cdx} 2$ is reported to induce ES cells to form mesoderm through BMP-induced differentiation [29], while Zic1 and Sall2 are zinc-finger transcription factors that play important roles in embryonic development [30, 31]. As before, real-time PCR verified these results (Fig. 2c).

To investigate the underlying mechanisms of AICAR supports ES cell self-renewal and pluripotency, the J1 ES cells were cultured in different conditions with or without AICAR, and the expression level of Oct4, Klf4, Nanog and Sox2 were examined by real-time PCR, cells cultured with LIF in normal medium were used as control. The real-time PCR result showed that their expression level was significantly decreased in the absence of LIF no matter with or without AICAR, comparing to cells cultured with LIF (Fig. 2d). This result indicated AICAR cannot maintain ES cell identity in the absence of LIF. However, compared with those exposed in $2 \mu \mathrm{M}$ RA alone, J1 ES cells cultured in the presence of AICAR showed significantly higher expression level of core pluripotency markers, no matter with or without LIF (Fig. 2e and 2f). Although the expression levels of these core pluripotency markers, excepting Oct4, were still lower than the LIF additional control (Fig. 2e and 2f). These results revealed that 
Shi et al.: AICAR Modulates mESCs Gene Expression

\begin{tabular}{lccccc}
\hline Terms & Count & Percent & P-Value & Fold Enrichment & Benjamini \\
\hline Pathways in cancer & 24 & 2.0 & $4.1 \mathrm{E}-2$ & 1.5 & $4.0 \mathrm{E}-1$ \\
MAPK signaling pathway & 21 & 1.7 & $3.2 \mathrm{E}-2$ & 1.6 & $3.8 \mathrm{E}-1$ \\
Endocytosis & 19 & 1.6 & $9.0 \mathrm{E}-3$ & 1.9 & $3.6 \mathrm{E}-1$ \\
Cell adhesion molecules (CAMs) & 18 & 1.5 & $1.2 \mathrm{E}-3$ & 2.4 & $1.6 \mathrm{E}-1$ \\
Lysosome & 12 & 1.0 & $2.9 \mathrm{E}-2$ & 2.1 & $4.2 \mathrm{E}-1$ \\
Antigen processing and presentation & 10 & 0.8 & $3.2 \mathrm{E}-2$ & 2.3 & $4.1 \mathrm{E}-1$ \\
Viral myocarditis & 10 & 0.8 & $3.8 \mathrm{E}-2$ & 2.2 & $3.0 \mathrm{E}-1$ \\
Glutathione metabolism & 9 & 0.7 & $3.3 \mathrm{E}-3$ & 2.9 & $2.1 \mathrm{E}-1$ \\
Type I diabetes mellitus & 9 & 0.7 & $1.1 \mathrm{E}-2$ & 2.2 & $4.2 \mathrm{E}-1$ \\
ECM-receptor interaction & 9 & 0.7 & $4.8 \mathrm{E}-2$ & 3.1 & $3.3 \mathrm{E}-1$ \\
Arginine and proline metabolism & 8 & 0.7 & $1.3 \mathrm{E}-2$ & 2.8 & $3.1 \mathrm{E}-1$ \\
Hedgehog signaling pathway & 8 & 0.7 & $1.5 \mathrm{E}-2$ & 2.8 & $3.6 \mathrm{E}-1$ \\
Graft-versus-host disease & 8 & 0.7 & $2.1 \mathrm{E}-2$ & 2.6 & $3.6 \mathrm{E}-1$ \\
Allograft rejection & 8 & 0.7 & $2.1 \mathrm{E}-2$ & 4.8 & $3.9 \mathrm{E}-1$ \\
Basal cell carcinoma & 7 & 0.6 & $4.9 \mathrm{E}-2$ & 3.3 & $3.2 \mathrm{E}-1$ \\
Steroid biosynthesis & 4 & 0.3 & $4.7 \mathrm{E}-2$ & 3.1 & $9.5 \mathrm{E}-1$ \\
TGF-beta signaling pathway & 10 & 1.0 & $3.2 \mathrm{E}-3$ & 4.9 & $8.9 \mathrm{E}-1$ \\
Cytosolic DNA-sensing pathway & 6 & 0.6 & $4.2 \mathrm{E}-2$ & & \\
Nitrogen metabolism & 4 & 0.4 & $4.5 \mathrm{E}-2$ & & \\
\hline KEGG pathway analysis of significantly up-regulated and down-regulated genes using the DAVID online program with the \\
thresholds of count $\geq 4$ and EASE $<0.05 .19$ pathway terms were identified. & \\
\hline
\end{tabular}

Table 1. KEGG pathway analysis of significantly upregulated $(1364)$ and downregulated $(1,119)$ genes in J1 mESCs after AICAR treatment

AICAR might play its role in antagonizing the action of RA-induced differentiation.

Taken together, our findings suggested that AICAR functions to maintain ES cell selfrenewal and inhibit differentiation via up-regulating the expression of pluripotency-related genes and down-regulating the differentiation-related genes, although there were some exceptions to this. As to its biological function, we found that AICAR cannot maintain ES cell pluripotency without LIF, but it can antagonize RA-induced differentiation.

\section{GO Term and KEGG Pathway Comparison of Transcripts Regulated by AICAR}

To overview the function of differently expressed genes identified by microarray analysis, we performed GO ontologies using the DAVID online program and identified 17 enriched terms associated with our gene list (Fig. 3a). The terms included in biology process (BP) FAT were regulation of transcription, regulation of RNA metabolic process, cell motion, embryonic organ development, negative regulation of cell differentiation, and neuron differentiation. Only plasma membrane part and plasma membrane were in cell component (CC) FAT. The identified terms in molecular function (MF) FAT were transcription factor activity, sequence-specific DNA binding, DNA binding, transcription regulator activity, calcium ion binding, zinc ion binding, and transition metal ion binding. This indicated that AICAR mainly functions by modulating gene transcription, affecting molecule binding, and influencing ES cell differentiation and embryonic development.

To better understand how AICAR influences the gene regulation pathways of J1 ES cells, we performed KEGG pathway analysis using the DAVID online program. With count $\geq 4$ and EASE $<0.05$ thresholds, we identified 19 pathway terms that were impacted by AICAR treatment (Table 1). As expected, these included pathways associated with ES cell self-renewal or differentiation, such as MAPK and TGF-beta signaling [32, 33]. Consistent with the view that AICAR is an activator of the AMPK signal pathway, pathways involved in energy metabolism were also identified, including arginine and proline metabolism, nitrogen metabolism and steroid biosynthesis. Furthermore, the hedgehog signaling pathway, which plays important roles in embryonic cells and during vertebrate embryonic development, was also influenced by the addition of AICAR. Finally, basal cell carcinoma, which is associated with the malfunction of the hedgehog signaling pathway, appeared to be affected by AICAR. 


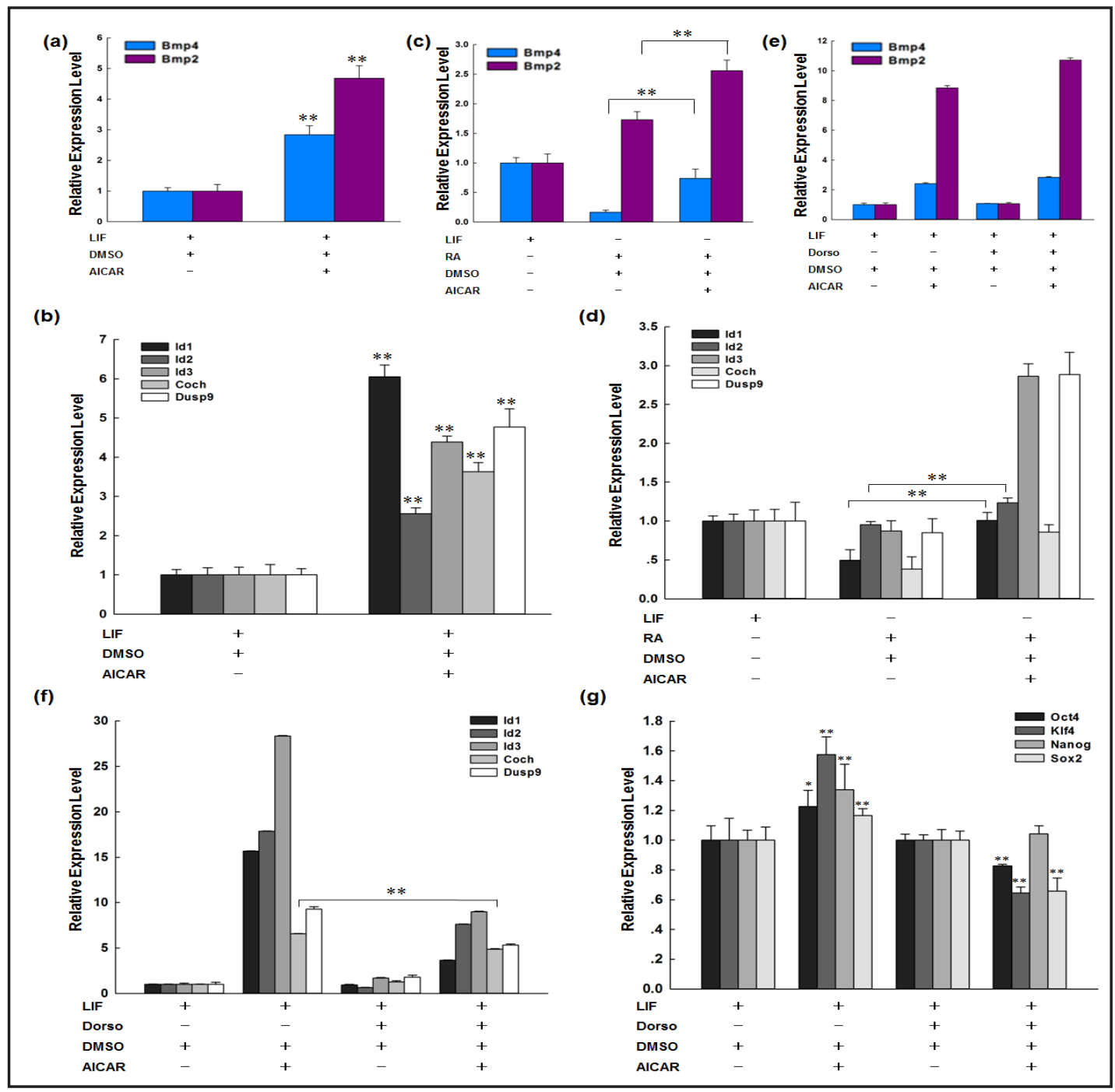

Fig. 4. AICAR function was associated with BMP signaling pathway. (a): J1 ES cells were cultured in LIF containing medium with or without AICAR for $24 \mathrm{~h}$, and Bmp2 and Bmp4 expression level were analyzed by real-time PCR using the comparative Ct method. GAPDH was used to normalize template levels. Data are presented as the mean \pm SD of three independent experiments. (b): J1 ES cells were cultured in LIF containing medium with or without AICAR for $24 \mathrm{~h}$, and expression of Id1, Id2, Id3, Coch, and Dusp9 were analyzed by real-time PCR using the comparative Ct method. GAPDH was used to normalize template levels. Data are presented as the mean \pm SD of three independent experiments. (c): Bmp2 and Bmp4 expression levels were examined by real-time PCR following treatment of $2 \mu \mathrm{M}$ RA or $2 \mu \mathrm{M}$ RA combined with $1 \mathrm{mM}$ AICAR, cells cultured in normal medium were used as control. GAPDH was used to normalize template levels. Data are presented as the mean \pm SD of three independent experiments. (d): Real-time PCR analysis of Id1, Id2, Id3, Coch and Dusp9 transcript level following the treatment of $2 \mu \mathrm{M}$ RA or $2 \mu \mathrm{M}$ RA combined with 1 mM AICAR, cells cultured in normal medium were used as control. GAPDH was used to normalize template levels. Data are presented as the mean \pm SD of three independent experiments. (e): J1 ES cells were cultured in the absence or presence of $5 \mu \mathrm{M}$ Dorso and with or without AICAR for $24 \mathrm{~h}$, the expression levels of Bmp2 and Bmp4 were examined by real-time PCR. GAPDH was used to normalize template levels. Data are presented as the mean \pm SD of three independent experiments. (f): J1 ES cells were cultured in the absence or presence of $5 \mu \mathrm{M}$ Dorso and with or without AICAR for $24 \mathrm{~h}$, the expression levels of Ids, Coch and Dusp9 were examined by real-time PCR. GAPDH was used to normalize template levels. Data are presented as the mean \pm SD of three independent experiments. (g): J1 ES cells were cultured in the absence or presence of $5 \mu \mathrm{M}$ Dorso and with or without AICAR for $24 \mathrm{~h}$, the expression levels of Oct4, Klf4, Nanog and Sox 2 were examined by real-time PCR. GAPDH was used to normalize template levels. Data are presented as the mean \pm SD of three independent experiments. $(*$ : $p<0.05 ; * *: \mathrm{p}<0.01)$. 


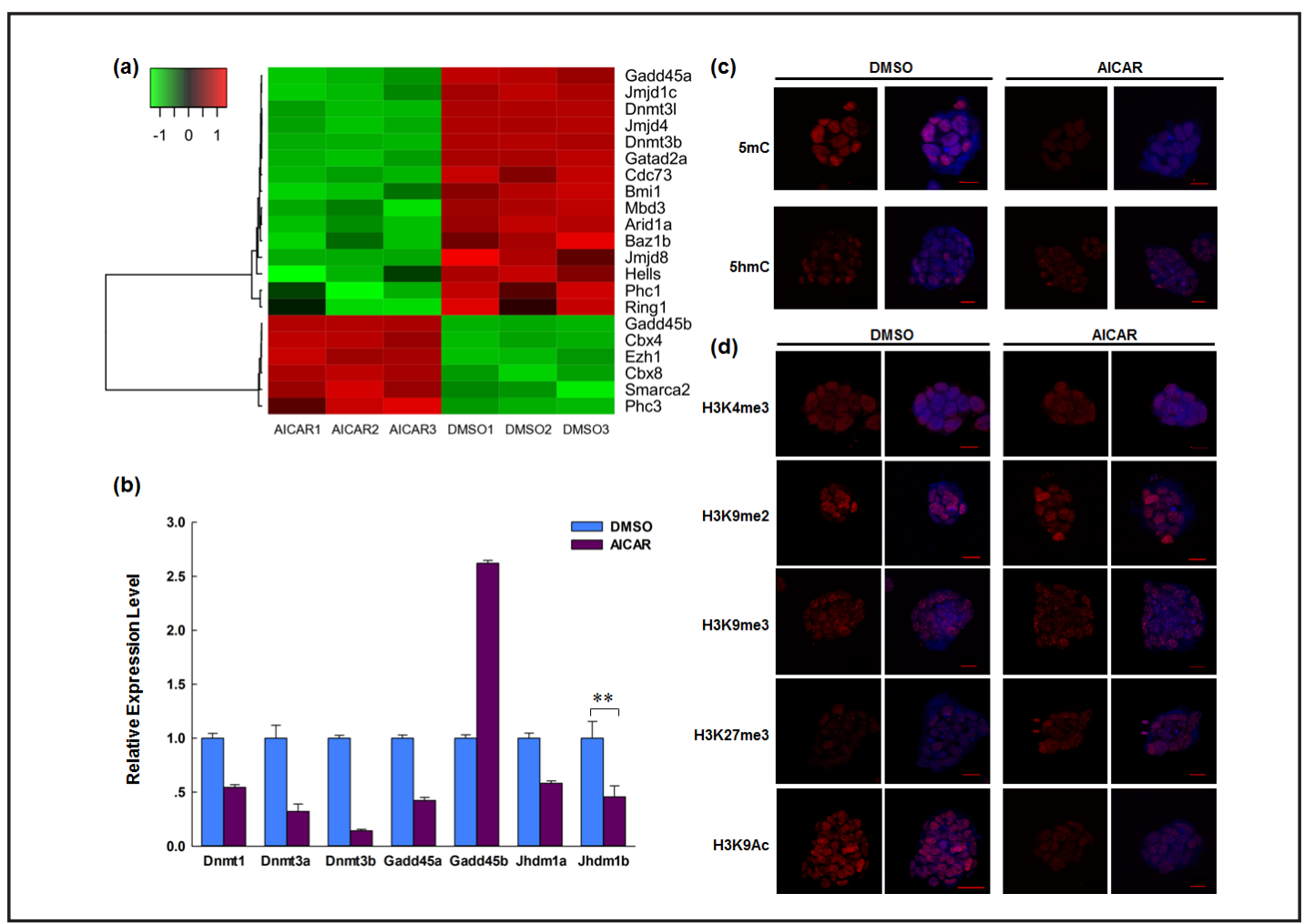

Fig. 5. Variations in the expression of J1 mESC epigenetic-related genes following AICAR treatment. (a): Expression pattern of representative epigenetic-associated genes identified by microarray data in J1 mESCs cultured with or without 1 mM AICAR. High expression levels are shown in green and low in red. (b): Representative epigenetic-related genes, differently expressed in the presence of $1 \mathrm{mM} \mathrm{AICAR}$, were validated by real-time PCR. J1 mESCs were cultured in medium containing serum and 1,000 U/ml LIF in the presence or absence of $1 \mathrm{mM}$ AICAR for $24 \mathrm{~h}$. Total RNA was isolated, and the relative expression level of genes determined by real-time PCR. GAPDH was used to normalize template levels. Data are presented as the mean \pm SD of three independent experiments. (c): Immunofluorescence staining of global DNA methylation level $5 \mathrm{mC}$ and $5 \mathrm{hmC}$ in J1 mESCs in the presence of 1,000 U/ml LIF with or without $1 \mathrm{mM}$ AICAR for $24 \mathrm{~h}$. Cell nuclei were stained by DAPI. Left columns represent antibody staining of each marker and right columns represent merged image with DAPI staining. Scale bars represent $20 \mu \mathrm{m}$. (d): Immunofluorescence staining of histone modifications, H3K4me3, H3K9me2, H3K9me3, H3K27me3, and H3K9Ac in J1 mESCs in the presence of $1,000 \mathrm{U} / \mathrm{ml}$ LIF with or without $1 \mathrm{mM}$ AICAR for $24 \mathrm{~h}$. Cell nuclei were stained by DAPI. Left columns represent antibody staining of each marker and right columns represent merged image with DAPI staining. Scale bars represent $20 \mu \mathrm{m}$. (*: $\mathrm{p}<0.05 ; * *: \mathrm{p}<0.01)$.

To verify KEGG pathway analysis and identify alternative key signaling pathways that may be influenced by AICAR, we performed a dual luciferase reporter assay using different pathway reporter vectors. Each pathway reporter vector has a specific cis-acting DNA binding sequence (enhancer element) located upstream from the firefly luciferase promoter,

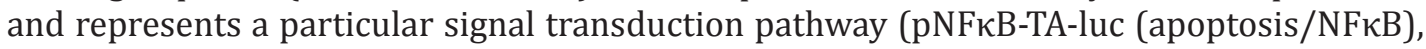
pISRE-TA-luc (JAK/STAT), pAP1(PMA)-TA-luc (PKC/MAPK), pMyc-TA-luc (cell proliferation), pAP1-TA-luc (JNK), pCRE-TA-luc (JNK/p38 and PKA), pGRE-TA-luc (glucocorticoid/ HSP90), pHSE-TA-luc (heat shock response), pSRE-TA-luc (MAPK/JNK)). We identified the induction of AICAR toward particular signaling pathways through monitoring the binding of transcription factors to enhancer elements. As a result, we found that AICAR negatively regulated apoptosis/NFkB, glucocorticoid/HSP90 and PKC pathway. On the other hand, JAK/ STAT and JNK signaling pathways were positively regulated by AICAR (Fig. 3b). Moreover, pMyc-TA-luc, which associated with cell proliferation, was down-regulated following the 
treatment of AICAR (Fig. 3b).

Taken together, our GO term and KEGG pathway analysis of differentially expressed genes indicated that, as a modulator in J1 ES cells, AICAR functions not only through directly impacting the transcription of pluripotency or differentiation-associated genes, but also through cross-talking between different signaling pathways to maintain ES cell self-renewal and totipotency.

The function of AICAR to J1 ES cells may associate with BMP signal pathway

In this study, we demonstrated that AICAR maintain J1 ES cell pluripotency in a LIF-dependent manner, it can maintain ES cell identity with LIF and inhibit RA-induced differentiation. It has been reported previously that BMP signaling pathway maintains ES cell self-renewal in collaboration with LIF through repressing neural differentiation. To investigate whether AICAR act through BMP signaling pathway, we treated J1 ES cells with AICAR or equal volume of DMSO for $24 \mathrm{~h}$, and expression level of BMP signaling-related genes and its downstream targets were determined by real-time PCR. The result showed that both Bmp2 and Bmp4 were up-regulated following the treatment of AICAR relative to LIF alone (Fig. 4a). In addition, the downstream targets of BMP signaling, including inhibitor of DNA binding proteins (Id; Id1, Id2 and Id3), coagulation factor C homolog (Coch) and dual specificity phosphatase 9 (Dusp9) were also increased by AICAR treatment (Fig. 4b). Among those BMP target genes, Ids are initially identified as critical downstream targets of BMP signaling pathway to block neural differentiation and sustain ES cell self-renewal [34]. Coch is recently described target of BMP pathway, and it can partially mimic Bmp4 to inhibit neural differentiation [35]. Dusp9 is a mediator of Bmp signaling and its up-regulation can steadily attenuate ERK activity [36].

To further investigate the potential association of AICAR and BMP signaling pathway, we examined the gene expression changes following treatment with RA or RA plus AICAR and without LIF, cells cultured in normal medium were used as control. The real-time PCR results showed that RA significantly decreased the expression of Bmp4, Id1, and Coch, while the level of Id2, Id3 and Dusp6 was almost unchanged. Bmp2, which was an exception, was increased following AICAR treatment (Fig. 4c and 4d). As expected, addition of AICAR compromised the function of RA and rescued the expression of Bmp2, Bmp4 and their target genes Ids, Coch and Dusp9 to some extent (Fig. 4c and 4d). However, the expression of Id1 and Coch levels did not increase above basal level in the presence of AICAR and RA, unlike the case with AICAR alone, that maybe the effect of RA. In addition, we had chosen a small molecule inhibitor of BMP pathway Dorsomorphin (Dorso) to investigate the possible role of AICAR in BMP pathway of J1 ES cells. We cultured J1 ES cells in the absence or presence of AICAR and with or without Dorso, the expression levels of Bmps, its downstream targets and core pluripotency markers were examined by real-time PCR. The results indicated that Dorso did not influence the expression of Bmp2 and Bmp4 significantly, that maybe the reason that Dorso was function downstream of Bmps (Fig. 4e). As to the downstream targets of Bmp4, their expression levels were decreased significantly, comparing AICAR plus Dorso sample with the sample of AICAR only (Fig. 4f), although their expressions still higher than those treated by Dorso only. Moreover, Dorso can compromise AICAR induced higher expression of core pluripotency markers (Fig. 4g).

Collectively, these data indicated that AICAR may partly act through enhancing BMP signaling pathway to inhibit RA-induced differentiation and enhance the expression of pluripotency-associated genes.

\section{AICAR Induces Epigenetic Modification Changes in J1 ES Cells}

Epigenetics is the study of heritable changes in gene expression and phenotype caused by mechanisms other than DNA sequence changes including DNA methylation, histone modification and regulations mediated by non-coding RNA. The ES cell genome appears to have its own unique epigenetic state that maintains self-renewal and rapidly induces variant transcription to initiate differentiation. 
In this study, we identified numerous genes participating in epigenetic modification through different pathways (Fig. 5a). These include Dnmt1, Dnmt3b, Dnmt31, Gadd45a and Gadd45b which participate in DNA methylation, and Arid1a, Baz1b, Smarca2, Ezh1, Bmi1, Phc1, Phc3, Ring1, Cdx4, Mbd3, and jumonji domain-containing proteins, which function in histone modification. Real-time PCR was performed to validate these microarray data and to identify other epigenetic-related genes that were not detected such as Dnmt3a, Jhdm1a and Jhdm 1 b (Fig. 5b). To our expected, our real-time results were consistent with the microarray data.

To investigate alterations of global epigenetic modifications in J1 ES cells, we performed immunofluorescence staining to examine epigenetic changes that involved in DNA methylation, for example 5-methylcytosine $(5 \mathrm{mC})$ and 5-hydroxymethyl cytosine $(5 \mathrm{hmC})$ (Fig. 5c), as well as those classified to histone modification (Fig. 5c), such as histone H3 lysine 4 tri-methylation (H3K4me3), and histone $\mathrm{H} 3$ lysine 9 acetylation (H3K9Ac), histone $\mathrm{H} 3$ lysine 4 di-methylation (H3K9me2), histone $\mathrm{H} 3$ lysine 9 tri-methylation (H3K9me3), and histone H3 lysine 27 tri-methylation (H3K27me3) (Fig. 5d). Presumably due to the decreased expression of Dnmt1, Dnmt3a and Dnmt3b, the global $5 \mathrm{mC}$ level was significantly reduced after the addition of $1 \mathrm{mM}$ AICAR. Although the expression of Gadd45a and Gadd45b was down-regulated and up-regulated, respectively, by AICAR, the $5 \mathrm{hmC}$ modification level in J1 ES cells was unchanged between cells cultured with or without AICAR. This may be because the expression of ten-eleven translocation (Tet) enzymes Tet1, Tet2 and Tet3, which convert 5-methylcytosine to 5-hydroxymethylcytosine, were also unchanged. The methylation of histone $\mathrm{H} 3$ at lysine 4 (H3K4) is usually catalyzed by methyltransferases of the SET1family (Set1a, Set1b, Mll1, Mll2, Mll3 and Mll4) and a constant expression level of SET-family genes may lead to invariable levels of H3K4me3. There was also no change in H3K9me2 and H3K9me3 levels, even though the expression level of G9a (also known as Ehmt2) showed a 1.30-fold change. Despite no change in Ezh2 expression levels, H3K27me3 modification increased following the addition of AICAR. This may be explained by the action of Ezh1 (FC = 1.94), which co-regulates H3K27me3 with Ezh2 and complements Ezh2 in stem cell maintenance $[37,38]$. As expected, H3K9Ac modification decreased after the addition of AICAR, probably because the expression level of many histone acetylases such as Kat5, Ncoa3, Kat2b, and Ep300 was down-regulated by AICAR, while the subunit of the histone deacetylase complex Mbd3 was up-regulated.

In addition, we identified 948 long intervening non-coding RNAs (lincRNAs) using the filter setting of FC $\geq 2$ or $\leq 0.5$ and $p \leq 0.01$ that were differentially transcribed between J1 ES cells treated with AICAR or DMSO. We then annotated two of these differentially transcribed lincRNAs by comparing them to linRNAdb. The first is linc1582 (chr8: 89996710-90049453 forward strand), which was increased by a fold-change 2.330, and the other is Tsix (chrX: 100626855-100680296 forward strand), which was decreased with a fold-change of 0.064 in J1 ES cells. Linc1582 is required for the normal expression of many pluripotency-associated genes such as Nanog, Oct4, Sox2, Klf4, and Zfp42 [39], so its up-regulation indicates a role in maintaining J1 ES cells pluripotency. Tsix is a negative regulator of X-inactive specific transcript (Xist) and is thought to regulate the early steps of X inactivation [40, 41]. Indeed, $\mathrm{X}$ chromosomes with down-regulated Tsix are inactivated more frequently than normal chromosomes [42].

Overall, our findings indicate that AICAR maintains J1 mESC self-renewal and pluripotency expression not only through regulating transcription factors, but also by altering epigenetic modifications such as DNA methylation, histone methylation, histone acetylation, chromatin remodeling, and non-coding RNA regulation.

\section{Discussion}

In the present study, we reported that AICAR can influence the expression of transcription factors that participate in ES cell fate decision and inhibit RA-induced differentiation. 
AICAR was also shown to function in many pathways including the BMP signaling, MAPK signaling and TGF- $\beta$ signaling pathways, which are associated with ES cell self-renewal and differentiation. Furthermore, AICAR alters the epigenetic modifications of J1 ES cell chromatin to maintain pluripotency.

The capacities to generate any cell type and to maintain an unlimited proliferative state are defining properties of ES cells, which derive from the inner cell mass of blastocysts [2]. As they are likely to be so useful in therapeutic applications including replacement therapies, and regenerative medicine of malignant injury and disease, it is essential to uncover the intrinsic and extrinsic mechanisms regulating their self-renewal and differentiation. This will also enable their efficient propagation and the orientation of differentiation modes.

Several transcription factors have been identified essential for ES cell self-renewal and pluripotency including Nanog, Oct4, Sox2, and Klf4. These and other factors compose three transcription networks: the core transcription network, the Myc-centered regulatory network and Polycomb complex factors [43]. Key pathways involved in ES cell modulation include the LIF/JAK-STAT signaling pathway, the TGF- $\beta$ signaling pathway, the MEK/ERK pathway, and the Wnt signaling pathway [4]. Small molecules that target particular members of these pathways have previously been explored for their role in ES cell maintenance and fate determination. For example, A-83-01 and SB431542 can function as TGF- $\beta$ inhibitors and repress ES cell differentiation [44, 45], PD0325901 and Pluripotin/SC1 can inhibit the MEK/ ERK pathway to enable ES cells to retain pluripotency and undergo self-renewal [7, 46], and CHIR99021, SB216763, and BIO are GSK3 inhibitors, which can up-regulate Wnt signaling and maintain ES cell stemness [8, 47]. These small molecules are relatively inexpensive and easy to transform. Furthermore, they can be delivered efficiently and we can finely control their dosage.

AMPK is a master metabolic modulator that can balance in vivo energy homeostasis and regulate several cellular processes including lipid metabolism, glucose transport, cell growth, polarity, migration, cytoskeletal dynamics, and autophagy [48]. It is activated in response to stresses that exhaust cellular ATP and generate large amounts of AMP. The small molecule AICAR is an analog of AMP that is capable of stimulating AMPK activity. In this study, we added AICAR to J1 ES cell culture medium and artificially depleted ATP so that AMPK would be activated, repress the energy consuming process and promote ATP generation. As expected, KEGG pathway analysis indicated many genes that have been modulated following the treatment of AICAR were involved in energy associated pathways, both energy producing and energy consuming processes. These pathways included glutathione metabolism, arginine and proline metabolism, steroid biosynthesis, and nitrogen metabolism, which includes degradative processes such as the urea cycle, as well as biosynthetic processes. This phenomenon suggests that an intracellular procedure that antagonizes energy exhaustion also represses the synthesis of proteins and stimulates their catabolism.

The addition of AICAR also led to a reduced expression of Cdk1 (Fig. 2a), which is essential for cell cycle progression and cell division. Researchers have previously shown that Cdk1 is required for maintaining ES cell self-renewal and its undifferentiated state [49], while Li and colleagues found that Cdk1 repressed ES cell differentiation into trophectoderm through enhancing Oct4 binding to Cdx2, which repressed Cdx2 expression [50]. In the present study, our microarray data revealed reduced expression of both Cdk1 and Cdx2 (Fig. $2 \mathrm{a}$ ), indicating that proteins other than $\mathrm{Cdk} 1$ can regulate $\mathrm{Cdx} 2$ expression and repress J1 ES cell differentiation. Although we observed that reduced Cdk1 expression always appeared during cell differentiation, this may not necessarily result in differentiation. Eomes is usually considered to be a differentiation-associated gene that is upregulated after AICAR treatment. As it is reported to be essential for mouse trophoblast formation and trophoblast cell self-renewal and differentiation, we presumed that this upregulation may be useful for the pluripotency of J1 ES cells cultured under feeder-free conditions [51].

KEGG pathway analysis indicated that AICAR significantly influences the TGF- $\beta$ signaling pathway, which is involved in many cellular biology processes including cell proliferation, apoptosis, in vivo homeostasis, and ES cell stemness maintenance and differentiation 
[52]. The TGF- $\beta$ superfamily contains numerous growth factors that are mainly classified into two groups: one comprises TGF $\beta$, Activin and Nodal, while the other consists of bone morphogenetic proteins (BMP) and growth differentiation factors (GDF). In our study, significantly differentially expressed genes that belong to the TGF- $\beta$ superfamily include Tgfb1, Lefty1, Lefty2, Smad2, Bmp2, Bmp4, and Smad6. Of these, Bmp2 and Bmp4 are involved in the Bmp signaling pathway which has an important role in human embryonic development and causes rapid differentiation of human (h)ESCs. By contrast, Bmp-mediated signaling in mESCs functions to sustain pluripotency and self-renewal of mESCs in combination with the LIF/JAK-STAT signal, which does not work in hESCs, through Id proteins but not TGF- $\beta$ [34, 53]. We have demonstrated the addition of AICAR not only increases the expression of Bmp2 and Bmp4, but also enhances their downstream targets expression, including Id1, Id2, Id3, Coch and Dusp9. This result indicated a potential mechanism of stemness maintenance of AICAR.

TGF- $\beta$ superfamily signaling pathway components Lefty 1 and Lefty 2 usually act by antagonizing nodal signaling, the epidermal growth factor-Cripto-1/FRL-1/Cryptic (EGFCFC) signaling pathway and other factors including activin and Bmp [52, 54, 55]. Lefty 1 and Lefty 2 are highly expressed in both human and mouse ES cells, but this expression does not decrease upon differentiation. Conversely, LIF withdrawal or the addition of RA cause increased expression of Lefty. Thus, Lefty may be at the crossroads of stemness and differentiation events, but its precise role in ESC fate determination is unknown [56]. The observed reduced expression of Lefty1 and Lefty2 in our study may be a result of antagonizing Bmp proteins or the decreased expression of Smad2, so may not directly relate to the ES cell fate decision.

ES cells have unique epigenetic modifications that allow them to maintain a pluripotent state and at the same time initiate expression of lineage-specific genes during cell differentiation [57]. This mechanism not only relies on the transcriptional network, but is also heavily dependent on modification of the chromatin within the cell by means of histone modification, DNA methylation and non-coding RNA regulation. In our study, a comparison of cells cultured in the presence or absence of AICAR revealed many differentially expressed genes involved in DNA or histone modification as well as numerous differentially transcribed lincRNA.

For example, Gadd45a and Gadd45b are involved in the base excision repair pathway, which is required by the TET-hydroxylase gene to promote active DNA demethylation, while Tet family genes have been demonstrated to play roles in ES cell maintenance and inner cell mass cell specification $[58,59]$. They also respond to environmental stresses by mediating activation of the JNK/p38 pathway, which was demonstrated in this study. Dnmt3b is a wellknown DNA methylase that is important for de novo DNA methylation, while Dnmt3l can stimulate DNMT3a activity and interact with HDAC1 to mediate transcription repression. It has been reported that Dnmt3a and Dnmt3b are dispensable for iPSCs in somatic cells [60, 61], and Dnmt3b also acts with Dnmt3a to methylate promoters of the pluripotency circuit factors including Nanog during germ layer differentiation. Jmjd1c, Jmjd4 and Jmjd8 are jumonji domain-containing proteins that are involved in histone demethylation. Shen and colleagues suggested that members of this family are required for the dynamic regulation of Polycomb complex activity, and thus fine-tune deposition of H3K27me3 and balance selfrenewal and differentiation of ES cells [62,63]. Bmi1, Phc1, Phc3, Ring1, Cdx4 and Cdx8 belong to the Polycomb repressive complexes, which mediate gene silencing by regulating chromatin structure through histone modification [64]. SWI/SNF (SWItch/Sucrose NonFermentable) is a nucleosome remodeling complex capable of destabilizing histone-DNA interactions to alter the position of the nucleosome along DNA. The differentially expressed genes Arid1a, Baz1b, and Smarca2 are also classified in this complex and the Arid1a mutant showed inhibited self-renewal in ES cells which differentiated into primitive endoderm-like cells. Mbd3 is a subunit of the nucleosome remodeling and histone deacetylation (NuRD) complex required for the proliferation and pluripotency of ES cells; an Mbd3 deficiency results in a failure of ES cells to undergo lineage development [65]. 
Shi et al.: AICAR Modulates mESCs Gene Expression

Thousands of lincRNAs have been identified in mammals, with large numbers being either critical for the maintenance of ES cell pluripotency or essential for repression of lineage-specific gene expression. We found that AICAR can promote the transcription of linc1582 and inhibit the transcription of Txis. Many other lincRNAs that were not annotated also showed significant changes after the addition of AICAR, but the roles of these remain to be elucidated.

In conclusion, the function of AICAR on ES cells is a sophisticated process, and the mechanism of how it maintains ES cell stemness warrants further study.

\section{Acknowledgments}

This work was supported by the National Major Project for Production of Transgenic Breeding (No. 2013ZX08007-004), the National High Technology Research and Development Program of China (863 Program) (No. 2011AA100303), and partly by a grant from the National Natural Science Foundation of China (No. 31172279). The authors would like to thank Qinhu Wang for excellent technical support.

\section{References}

1 Evans MJ, Kaufman MH: Establishment in culture of pluripotential cells from mouse embryos. Nature 1981;292:154-156.

- Brook FA, Gardner RL: The origin and efficient derivation of embryonic stem cells in the mouse. Proc Natl Acad Sci U S A 1997;94:5709-5712.

-3 Suda Y, Suzuki M, Ikawa Y, Aizawa S: Mouse embryonic stem cells exhibit indefinite proliferative potential. J Cell Physiol 1987;133:197-201.

4 Loebel DAF, Watson CM, De Young RA, Tam PPL: Lineage choice and differentiation in mouse embryos and embryonic stem cells. Dev Biol 2003;264:1-14.

5 Li W, Ding S: Small molecules that modulate embryonic stem cell fate and somatic cell reprogramming. Trends Pharmacol Sci 2010;31:36-45.

6 Ao A, Hao J, Hong CC: Regenerative chemical biology: Current challenges and future potential. Chem Biol 2011;18:413-424.

7 Chen S, Do JT, Zhang Q, Yao S, Yan F, Peters EC, Scholer HR, Schultz PG, Ding S: Self-renewal of embryonic stem cells by a small molecule. Proc Natl Acad Sci USA 2006;103:17266-17271.

-8 Ye S, Tan L, Yang R, Fang B, Qu S, Schulze EN, Song H, Ying Q, Li P: Pleiotropy of glycogen synthase kinase-3 inhibition by chir99021 promotes self-renewal of embryonic stem cells from refractory mouse strains. PLoS One 2012; 7:e35892.

-9 Sato N, Meijer L, Skaltsounis L, Greengard P, Brivanlou AH: Maintenance of pluripotency in human and mouse embryonic stem cells through activation of wnt signaling by a pharmacological gsk-3-specific inhibitor. Nat Med 2004;10:55-63.

10 Zhu S, Li W, Zhou H, Wei W, Ambasudhan R, Lin T, Kim J, Zhang K, Ding S: Reprogramming of human primary somatic cells by oct4 and chemical compounds. Cell Stem Cell 2010;7:651-655.

11 Chambers SM, Fasano CA, Papapetrou EP, Tomishima M, Sadelain M, Studer L: Highly efficient neural conversion of human es and ips cells by dual inhibition of smad signaling. Nat Biotechnol 2009;27:275-280.

12 Zang Y, Yu LF, Nan FJ, Feng LY, Li J: Amp-activated protein kinase is involved in neural stem cell growth suppression and cell cycle arrest by 5-aminoimidazole-4-carboxamide-1-beta-d-ribofuranoside and glucose deprivation by down-regulating phospho-retinoblastoma protein and cyclin d. J Biol Chem 2009;284:61756184.

13 Taub M, Cutuli F: Activation of amp kinase plays a role in the increased apoptosis in the renal proximal tubule in cystinosis. Biochem Biophys Res Commun 2012;426:516-521.

14 Gaidhu MP, Frontini A, Hung S, Pistor K, Cinti S, Ceddia RB: Chronic amp-kinase activation with aicar reduces adiposity by remodeling adipocyte metabolism and increasing leptin sensitivity. J Lipid Res 2011;52:17021711. 
Shi et al.: AICAR Modulates mESCs Gene Expression

15 Jorgensen SB, Honeyman J, Oakhill JS, Fazakerley D, Stockli J, Kemp BE, Steinberg GR: Oligomeric resistin impairs insulin and aicar-stimulated glucose uptake in mouse skeletal muscle by inhibiting glut4 translocation. Am J Physiol Endocrinol Metab 2009;297:E57-66.

-16 Oliveira SM, Zhang YH, Solis RS, Isackson H, Bellahcene M, Yavari A, Pinter K, Davies JK, Ge Y, Ashrafian H, Walker JW, Carling D, Watkins H, Casadei B, Redwood C: Amp-activated protein kinase phosphorylates cardiac troponin i and alters contractility of murine ventricular myocytes. Circ Res 2012;110:1192-1201.

17 Kubota S, Ozawa Y, Kurihara T, Sasaki M, Yuki K, Miyake S, Noda K, Ishida S, Tsubota K: Roles of amp-activated protein kinase in diabetes-induced retinal inflammation. Invest Ophthalmol Vis Sci 2011;52:9142-9148.

18 Chae HD, Lee MR, Broxmeyer HE: 5-aminoimidazole-4-carboxyamide ribonucleoside induces g(1)/s arrest and nanog downregulation via p53 and enhances erythroid differentiation. Stem Cells 2012;30:140-149.

19 Adamo L, Zhang Y, Garcia-Cardena G: Aicar activates the pluripotency transcriptional network in embryonic stem cells and induces klf4 and klf2 expression in fibroblasts. BMC Pharmacol 2009;9:2.

20 Pall E, Groza I, Cenariu M, Soritau O, Gocza E, Tomuleasa C: Establishment of an embryonic stem cell line from blastocyst stage mouse embryos. Rom J Morphol Embryol 2011;52:1005-1010.

21 Loh YH, Wu Q, Chew JL, Vega VB, Zhang W, Chen X, Bourque G, George J, Leong B, Liu J, Wong KY, Sung KW, Lee CW, Zhao XD, Chiu KP, Lipovich L, Kuznetsov VA, Robson P, Stanton LW, Wei CL, Ruan Y, Lim B, Ng HH: The oct4 and nanog transcription network regulates pluripotency in mouse embryonic stem cells. Nat Genet 2006;38:431-440.

22 Matoba R, Niwa H, Masui S, Ohtsuka S, Carter MG, Sharov AA, Ko MS: Dissecting oct3/4-regulated gene networks in embryonic stem cells by expression profiling. PLoS One 2006;1:e26.

-23 Zhang P, Andrianakos R, Yang Y, Liu C, Lu W: Kruppel-like factor 4 (klf4) prevents embryonic stem (es) cell differentiation by regulating nanog gene expression. J Biol Chem 2010;285:9180-9189.

24 Jauch R, Ng CK, Saikatendu KS, Stevens RC, Kolatkar PR: Crystal structure and DNA binding of the homeodomain of the stem cell transcription factor nanog. J Mol Biol 2008;376:758-770.

25 Ma Z, Swigut T, Valouev A, Rada-Iglesias A, Wysocka J: Sequence-specific regulator prdm14 safeguards mouse escs from entering extraembryonic endoderm fates. Nat Struct Mol Biol 2011;18:120-127.

-26 Zhang X, Zhang J, Wang T, Esteban MA, Pei D: Esrrb activates oct4 transcription and sustains self-renewal and pluripotency in embryonic stem cells. J Biol Chem 2008;283:35825-35833.

27 Jang H, Kim TW, Yoon S, Choi SY, Kang TW, Kim SY, Kwon YW, Cho EJ, Youn HD: O-glcnac regulates pluripotency and reprogramming by directly acting on core components of the pluripotency network. Cell Stem Cell 2012;11:62-74.

28 Chen J, Lu Z, Cheng D, Peng S, Wang H: Isolation and characterization of porcine amniotic fluid-derived multipotent stem cells. PLoS One 2011;6:e19964.

29 Bernardo AS, Faial T, Gardner L, Niakan KK, Ortmann D, Senner CE, Callery EM, Trotter MW, Hemberger M, Smith JC, Bardwell L, Moffett A, Pedersen RA: Brachyury and cdx2 mediate bmp-induced differentiation of human and mouse pluripotent stem cells into embryonic and extraembryonic lineages. Cell Stem Cell 2011;9:144-155.

30 Merzdorf CS, Sive HL: The zic1 gene is an activator of wnt signaling. Int J Dev Biol 2006;50:611-617.

- 31 Sato A, Matsumoto Y, Koide U, Kataoka Y, Yoshida N, Yokota T, Asashima M, Nishinakamura R: Zinc finger protein sall2 is not essential for embryonic and kidney development. Mol Cell Biol 2003;23:62-69.

-32 Watabe T, Miyazono K: Roles of tgf-beta family signaling in stem cell renewal and differentiation. Cell Res 2009;19:103-115.

33 Dalton S: Signaling networks in human pluripotent stem cells. Curr Opin Cell Biol 2013;25:241-246.

-34 Ying Q-L, Nichols J, Chambers I, Smith A: Bmp induction of id proteins suppresses differentiation and sustains embryonic stem cell self-renewal in collaboration with stat3. Cell 2003;115:281-292.

-35 Zhang J, Fei T, Li Z, Zhu G, Wang L, Chen YG: Bmp induces cochlin expression to facilitate self-renewal and suppress neural differentiation of mouse embryonic stem cells. J Biol Chem 2013;288:8053-8060.

-36 Li Z, Fei T, Zhang J, Zhu G, Wang L, Lu D, Chi X, Teng Y, Hou N, Yang X, Zhang H, Han JD, Chen YG: Bmp4 signaling acts via dual-specificity phosphatase 9 to control erk activity in mouse embryonic stem cells. Cell Stem Cell 2012;10:171-182.

-37 Shen X, Liu Y, Hsu YJ, Fujiwara Y, Kim J, Mao X, Yuan GC, Orkin SH: Ezh1 mediates methylation on histone h3 lysine 27 and complements ezh2 in maintaining stem cell identity and executing pluripotency. Mol Cell 2008;32:491-502.

-38 Ezhkova E, Lien WH, Stokes N, Pasolli HA, Silva JM, Fuchs E: Ezh1 and ezh2 cogovern histone h3k27 trimethylation and are essential for hair follicle homeostasis and wound repair. Genes Dev 2011;25:485498.

-39 Guttman M, Donaghey J, Carey BW, Garber M, Grenier JK, Munson G, Young G, Lucas AB, Ach R, Bruhn L, Yang X, Amit I, Meissner A, Regev A, Rinn JL, Root DE, Lander ES: Lincrnas act in the circuitry controlling pluripotency and differentiation. Nature 2011;477:295-300. 
Shi et al.: AICAR Modulates mESCs Gene Expression

40 Stavropoulos N, Lu N, Lee JT: A functional role for tsix transcription in blocking xist rna accumulation but not in X-chromosome choice. Proc Natl Acad Sci U S A 2001;98:10232-10237.

41 Clerc P, Avner P: New lessons from random x-chromosome inactivation in the mouse. J Mol Biol 2011;409:6269.

42 Lee JT, Davidow LS, Warshawsky D: Tsix, a gene antisense to xist at the x-inactivation centre. Nat Genet 1999;21:400-404.

43 Kim J, Woo AJ, Chu J, Snow JW, Fujiwara Y, Kim CG, Cantor AB, Orkin SH: A myc network accounts for similarities between embryonic stem and cancer cell transcription programs. Cell 2010;143:313-324.

44 Li W, Wei W, Zhu S, Zhu J, Shi Y, Lin T, Hao E, Hayek A, Deng H, Ding S: Generation of rat and human induced pluripotent stem cells by combining genetic reprogramming and chemical inhibitors. Cell Stem Cell 2009;4:16-19.

45 Lin T, Ambasudhan R, Yuan X, Li W, Hilcove S, Abujarour R, Lin X, Hahm HS, Hao E, Hayek A, Ding S: A chemical platform for improved induction of human ipscs. Nat Methods 2009;6:805-808.

46 Ying QL, Wray J, Nichols J, Batlle-Morera L, Doble B, Woodgett J, Cohen P, Smith A: The ground state of embryonic stem cell self-renewal. Nature 2008;453:519-523.

47 Lange C, Mix E, Frahm J, Glass A, Muller J, Schmitt O, Schmole AC, Klemm K, Ortinau S, Hubner R, Frech MJ, Wree A, Rolfs A: Small molecule gsk-3 inhibitors increase neurogenesis of human neural progenitor cells. Neurosci Lett 2011;488:36-40.

48 Ma Y, Yang F, Wang Y, Du Z, Liu D, Guo H, Shen J, Peng H: Camkkbeta is involved in amp-activated protein kinase activation by baicalin in lkb1 deficient cell lines. PLoS One 2012;7:e47900.

-49 Zhang WW, Zhang XJ, Liu HX, Chen J, Ren YH, Huang DG, Zou XH, Xiao W: Cdk1 is required for the self-renewal of mouse embryonic stem cells. J Cell Biochem 2011;112:942-948.

50 Li L, Wang J, Hou J, Wu Z, Zhuang Y, Lu M, Zhang Y, Zhou X, Li Z, Xiao W, Zhang W: Cdk1 interplays with oct4 to repress differentiation of embryonic stem cells into trophectoderm. FEBS Lett 2012;586:4100-4107.

-51 Russ AP, Wattler S, Colledge WH, Aparicio SA, Carlton MB, Pearce JJ, Barton SC, Surani MA, Ryan K, Nehls $\mathrm{MC}$, Wilson V, Evans MJ: Eomesodermin is required for mouse trophoblast development and mesoderm formation. Nature 2000;404:95-99.

52 Puceat M: Tgfbeta in the differentiation of embryonic stem cells. Cardiovasc Res 2007;74:256-261.

53 Niwa H, Burdon T, Chambers I, Smith A: Self-renewal of pluripotent embryonic stem cells is mediated via activation of stat3. Genes Dev 1998;12:2048-2060.

54 Sakuma R, Ohnishi Yi Y, Meno C, Fujii H, Juan H, Takeuchi J, Ogura T, Li E, Miyazono K, Hamada H: Inhibition of nodal signalling by lefty mediated through interaction with common receptors and efficient diffusion. Genes Cells 2002;7:401-412.

55 Cheng SK, Olale F, Brivanlou AH, Schier AF: Lefty blocks a subset of tgfbeta signals by antagonizing egf-cfc coreceptors. PLoS Biol 2004;2:E30.

56 Tabibzadeh S, Hemmati-Brivanlou A: Lefty at the crossroads of "stemness" and differentiative events. Stem Cells 2006;24:1998-2006.

57 Christophersen NS, Helin K: Epigenetic control of embryonic stem cell fate. J Exp Med 2010;207:2287-2295.

58 Thaler R, Spitzer S, Karlic H, Klaushofer K, Varga F: Dmso is a strong inducer of DNA hydroxymethylation in pre-osteoblastic mc3t3-e1 cells. Epigenetics 2012;7:635-651.

59 Freudenberg JM, Ghosh S, Lackford BL, Yellaboina S, Zheng X, Li R, Cuddapah S, Wade PA, Hu G, Jothi R: Acute depletion of tet1-dependent 5-hydroxymethylcytosine levels impairs lif/stat3 signaling and results in loss of embryonic stem cell identity. Nucleic Acids Res 2012;40:3364-3377.

60 Trowbridge JJ, Orkin SH: Dnmt3a silences hematopoietic stem cell self-renewal. Nat Genet 2012;44:13-14.

61 Pawlak M, Jaenisch R: De novo DNA methylation by dnmt3a and dnmt3b is dispensable for nuclear reprogramming of somatic cells to a pluripotent state. Genes Dev 2011;25:1035-1040.

62 Peng JC, Valouev A, Swigut T, Zhang J, Zhao Y, Sidow A, Wysocka J: Jarid2/jumonji coordinates control of prc2 enzymatic activity and target gene occupancy in pluripotent cells. Cell 2009;139:1290-1302.

63 Shen X, Kim W, Fujiwara Y, Simon MD, Liu Y, Mysliwiec MR, Yuan GC, Lee Y, Orkin SH: Jumonji modulates polycomb activity and self-renewal versus differentiation of stem cells. Cell 2009;139:1303-1314.

64 So AY, Jung JW, Lee S, Kim HS, Kang KS: DNA methyltransferase controls stem cell aging by regulating bmi1 and ezh2 through micrornas. PLoS One 2011;6:e19503.

65 Kaji K, Caballero IM, MacLeod R, Nichols J, Wilson VA, Hendrich B: The nurd component mbd3 is required for pluripotency of embryonic stem cells. Nat Cell Biol 2006;8:285-292. 